\title{
COMMUNICATION
}

Cite this: DOI: 10.1039/xoxxooooox

Accepted ooth January 2012

DOI: 10.1039/xoxxooooox

www.rsc.org/

\section{Charge-Carrier Dynamics in Vapour-Deposited Films of the Organolead Halide Perovskite $\mathrm{CH}_{3} \mathrm{NH}_{3} \mathrm{PbI}_{3-x} \mathrm{Cl}_{x} \uparrow$}

\author{
Christian Wehrenfennig, Mingzhen Liu, Henry J. Snaith, Michael B. Johnston and \\ Laura M. Herz*
}

We determine high charge carrier mobilities $\geq 33 \mathrm{~cm}^{2} \mathrm{~V}^{-1} \mathrm{~s}^{-1}$ and bi-molecular recombination rates about five orders of magnitude below the prediction of Langevin's model for vapour-deposited $\mathrm{CH}_{3} \mathrm{NH}_{3} \mathrm{PbI}_{3-\mathrm{x}} \mathrm{Cl}_{\mathrm{x}}$ using ultrafast $\mathrm{THz}$ spectroscopy. At charge carrier densities below $\sim 10^{17} \mathrm{~cm}^{-3}$ intrinsic diffusion lengths are shown to approach 3 microns, limited by slow mono-molecular decay processes.

Vapour deposition is a well-established technique in the commercial fabrication of optoelectronic devices, offering a wide range of benefits in terms of cost, scalability, environmental impact and high film quality. ${ }^{123}$ An exciting recent example has been the demonstration of thin-film organolead halide perovskites solar cells from a simple dualsource evaporation protocol. ${ }^{4}$ These cells were found to exhibit high power conversion efficiencies of up to $15.4 \%$ based on a simple planar heterojunction device design. In comparison to solution processing techniques, vapour deposition allows for high film homogeneity, avoiding problems caused by dewetting and subsequent pin-hole formation. ${ }^{5}$ In addition, vapour deposition greatly simplifies the fabrication of tandem cells comprising material layers of complementary absorption spectra, obviating the need for orthogonal solvents and more elaborate crosslinking schemes. Such tandem cells can potentially surpass the theoretical Shockley-Queisser limit for the power conversion efficiency of $33 \%$ for single-junction cells. ${ }^{6}$ For cells based on $\mathrm{CH}_{3} \mathrm{NH}_{3} \mathrm{PbI}_{3-\mathrm{x}} \mathrm{Cl}_{\mathrm{x}}$, this concept is particularly attractive since the bandgap energy of near $1.6 \mathrm{eV}$ (ref. ${ }^{7}$ ) is larger than the optimum value, leaving a wide spectral range in the near-infrared unused.

The past year has seen a tremendous surge of research on organo-lead halide perovskites after the first high-efficiency solid state solar cells based on these materials were reported. ${ }^{89}$ Data on many fundamental material properties of these materials is emerging and the understanding of the principal optoelectronic processes governing photovoltaic operation has increased significantly. ${ }^{10} 11$ Apart from the intrinsic material properties that render organolead halide perovskites so suitable as absorbers in solar cell, non-intrinsic factors such as morphology, ${ }^{12} 13$ crystallinity and stoichiometry ${ }^{7} 14$ have been found to have considerable effects on device performance. ${ }^{15}$ However, past studies have almost exclusively been performed on materials prepared through solution-based deposition protocols. Given the attractive benefits of vapour deposition, further knowledge on relevant properties for photovoltaic operation of these films is therefore urgently required. Such information may also reveal which particular aspects of the charge generation and recombination processes are inherent to a given material, and which elements may be further tuned through suitable alterations of the fabrication protocol.

In this letter, we report recombination lifetimes and carrier mobility in vapour deposited films of the leadhalide perovskite $\mathrm{CH}_{3} \mathrm{NH}_{3} \mathrm{PbI}_{3-x} \mathrm{Cl}_{x}$ as two key properties for photovoltaic operation. Materials were made using a dual source evaporation protocol $\left(\mathrm{CH}_{3} \mathrm{NH}_{3} \mathrm{I} / \mathrm{PbCl}_{2}\right)$ in the same way as previously reported. ${ }^{4}$ By measuring the time-resolved, photoinduced $\mathrm{THz}$ conductivity in these films, we monitor the charge-carrier dynamics over a wide range of excitation densities and extract the recombination rate constants. In addition, we establish a remarkably high charge-carrier mobility in excess of $33 \mathrm{~cm}^{2} \mathrm{~V}^{-1} \mathrm{~s}^{-1}$. Taken together, our results show that these vapour deposited films allow for charge-carrier diffusion length in excess of several microns under typical solar-cell operating conditions. We demonstrate that these long diffusion lengths result from a combination of both, low bimolecular electronhole recombination rates far below the prediction of the Langevin model, and slow effective trap-mediated recombination of charge carriers.

Ultrafast optical-pump-THz-probe spectroscopy as a timeresolved, contactless conductivity probe has previously been successfully employed to investigate the dynamics of photoexcited excitons and free charges. ${ }^{16} 17 \quad 1819$ For the interpretation of the photoinduced $\mathrm{THz}$ response of $\mathrm{CH}_{3} \mathrm{NH}_{3} \mathrm{PbI}_{3-x} \mathrm{Cl}_{x}$ it is first necessary to consider which optically excited species may couple to the $\mathrm{THz}$ probe pulse. Reported exciton binding energies for the closely related organo-lead halide perovskite $\mathrm{CH}_{3} \mathrm{NH}_{3} \mathrm{PbI}_{3}$ range between 19 
and $50 \mathrm{meV},{ }^{20212223}$ so a coexistence of both, free charges and excitons in thermal equilibrium at room temperature seems possible. However, D'Innocenzo et al. have recently shown that for low charge carrier concentrations, free charges are expected to dominate the photoexcited population with excitons only forming a minority species. ${ }^{23}$ In order to determine the type of excitations the $\mathrm{THz}$ probe pulse interacts with, we proceed to analyze the frequency spectrum of the photoinduced $\mathrm{THz}$ response.

Figure 1 shows $\mathrm{THz}$ photoinduced absorption spectra for $\mathrm{CH}_{3} \mathrm{NH}_{3} \mathrm{PbI}_{3-x} \mathrm{Cl}_{x}$ recorded at 20 ps and 2 ns pump-probe delay. The spectral shape does not change with time after excitation and displays an essentially flat real part and only a very small imaginary component. Such a response is consistent with the predictions of a standard Drude conductivity model for free carriers at frequencies much lower than the relaxation rate. ${ }^{19} 17$ We therefore conclude that the observed OPTP signal predominantly arises from the interaction of the $\mathrm{THz}$ probe with free charge carriers. From this observation alone, we cannot necessarily rule out the presence of excitons, as the $\mathrm{THz}$ probe may simply not interact with any present excitonic species. However, as we discuss in more detail below, there is mounting evidence that at room temperature, free charges are predominantly created in these materials. ${ }^{24}{ }^{25}{ }^{23} \mathrm{We}$ therefore expect the $\mathrm{THz}$ photoconductivity response to be representative of the majority of free charge created by the visible light pulse.

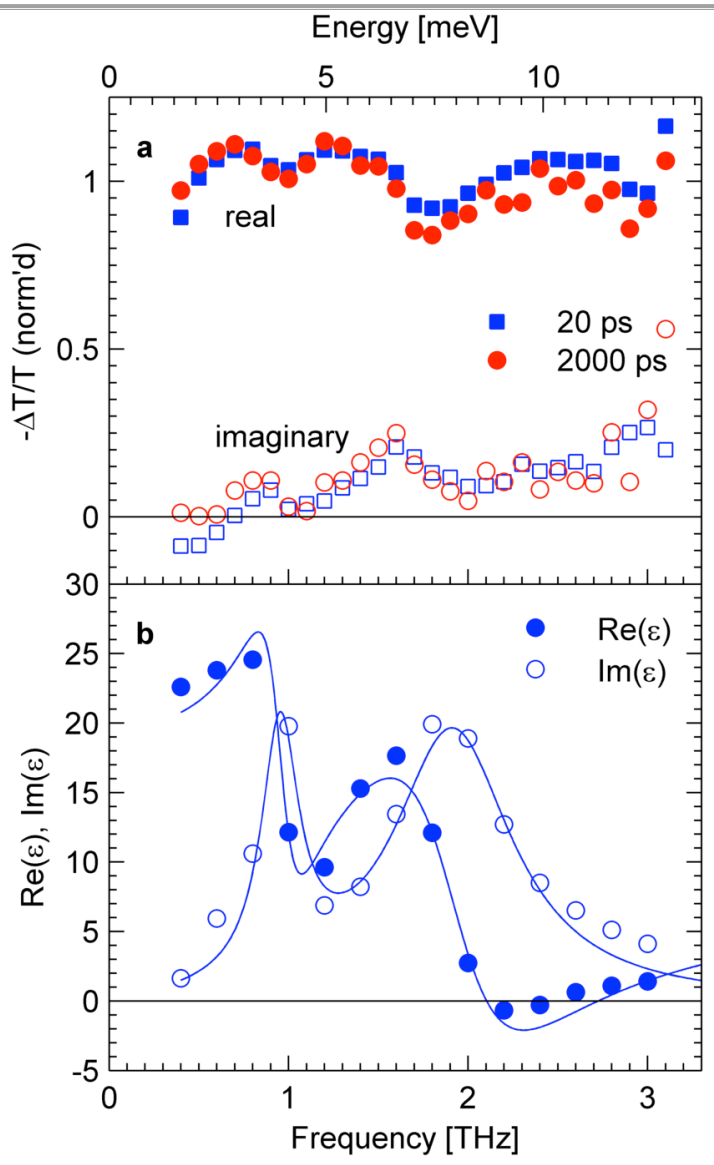

Figure 1: (a) Normalized, complex $\mathrm{THz}$ photoinduced absorption spectra of vapour-deposited $\mathrm{CH}_{3} \mathrm{NH}_{3} \mathrm{Pbl}_{3-x} \mathrm{Cl}_{x}$ (closed symbols - real parts, open symbols - imaginary parts) for two different time-delays (20 ps and $2000 \mathrm{ps}$ ) after excitation at $550 \mathrm{~nm}(2.25 \mathrm{eV})$ with pulse fluence $280 \mu \mathrm{Jm}^{-2}$. The slight drop-off towards low frequencies $(<0.5 \mathrm{THz})$ is due to an artifact resulting from the large diffraction limited beam waist for these frequency components approaching the diameter of the optical pump beam. (b) THz dark permittivity spectra (no photoexcitation), fitted with the output of a Lorentz Oscillator model including two resonances, as described in the main text and in detail in ESI.

We also note that the $\mathrm{THz}$ spectra show no substantial signs of disorder-induced scattering phenomena, which generally cause a drop of the real part and the emergence of a non-zero imaginary part of the conductivity towards low frequencies. ${ }^{2627}$ This is in agreement with the existence of relatively large crystalline domains previously reported for these vapourdeposited thin films. ${ }^{4}$ We find that the THz spectra also show some weak additional features centered at around $1 \mathrm{THz}$ and $2 \mathrm{THz}$. To examine the origin of these features, we also measured the $\mathrm{THz}$ permittivity spectrum in absence of photoexcitation, which is shown in Figure $1 \mathrm{~b}$. These spectra show two clear resonances in the same spectral regions for which weak features are observed in the photoinduced conductivity spectra. We are able to perform simultaneous fits to both real and imaginary parts of the dark permittivity using a standard Lorentz Oscillator model including two resonances (see ESI for full details) yielding resonance frequencies $f_{0}{ }^{(1)}=0.96 \mathrm{THz}$ and $f_{0}^{(2)}=1.96 \mathrm{THz}$. A mode centered at a wavenumber of $62 \mathrm{~cm}^{-1}(1.86 \mathrm{THz})$ has previously been observed in Raman spectra of $\mathrm{CH}_{3} \mathrm{NH}_{3} \mathrm{PbCl}_{3}$ (ref. ${ }^{28}$ ) and $\mathrm{CH}_{3} \mathrm{NH}_{3} \mathrm{PbI}_{3}$ (ref. ${ }^{29}$ ), and was assigned to vibrational modes associated with bending and stretching of the lead-halide bonds. The features observable in the photoconductivity spectra therefore most likely arise from a photoinduced modulation of such lead-halide sub-lattice phonon modes superimposed on the Drude conductivity response. We note that this scenario suggests that charge carriers couple effectively to lattice vibrations in $\mathrm{CH}_{3} \mathrm{NH}_{3} \mathrm{PbI}_{3-x} \mathrm{Cl}_{x}$. In agreement with these findings, phonon coupling has recently been suggested to induce the broadening of the emission from these materials. ${ }^{30}$ Less recent studies on metal halide crystals have generally emphasized the importance of lattice distortion around charge carriers in such systems, with coupling to e.g. longitudinal phonons driving a polaronic relaxation around a photoinduced charge pair. ${ }^{31}$ Hence polaronic effects may have to be taken into account when the electronic properties of this material are considered.

We proceed to investigate the charge carrier recombination dynamics in vapour deposited $\mathrm{CH}_{3} \mathrm{NH}_{3} \mathrm{PbI}_{3-x} \mathrm{Cl}_{x}$ films through analysis of data from time-dependent optical-pump-THz probe experiments. Figure 2 shows $\mathrm{THz}$ absorption transients after photoexcitation at $550 \mathrm{~nm}(2.25 \mathrm{eV})$ for a range of excitation fluences between 6 and $188 \mu \mathrm{J} \mathrm{cm} \mathrm{cm}^{-2}$. The relative $\mathrm{THz}$ absorption change is directly proportional to the photoinduced conductivity of the film (see Ref. ${ }^{32}$ and ESI) and therefore yields information on the product of charge carrier density and mobility. Given the increasingly fast decay dynamics at elevated excitation fluences, we model the time-dependence of the transients in terms of a free-charge population decay, neglecting possible changes of carrier mobility on the investigated timescale. 


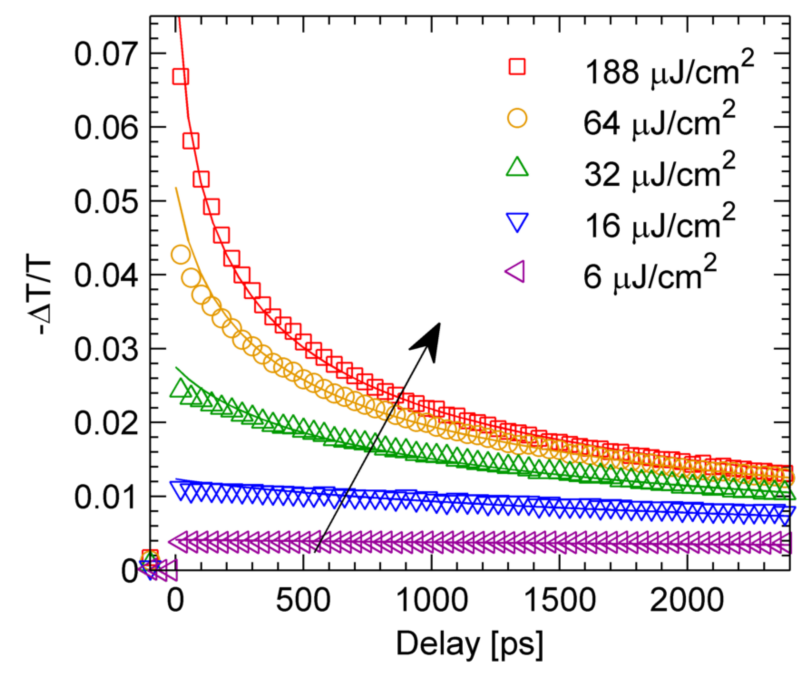

Figure 2: $\mathrm{THz}$ photoinduced absorption transients of vapourdeposited thin films of $\mathrm{CH}_{3} \mathrm{NH}_{3} \mathrm{Pbl}_{3-x} \mathrm{Cl}_{x}$ at excitation fluences between 6 and $188 \mu \mathrm{J} \mathrm{cm}^{-2}$ and for excitation wavelength $550 \mathrm{~nm}(2.25 \mathrm{eV})$. Solid lines represent fits with a $2^{\text {nd }}$ - and $3^{\text {rd }}-$ order decay function with globally optimized rate constants (equal for all fluences) as described in the text and in ESI.

We first extract a value for the effective charge-carrier mobility $\varphi \mu$ at $\mathrm{THz}$ frequencies for the material from the photoinduced $\mathrm{THz}$ transmission change extrapolated to zero pump-probe-delay under knowledge of the initially absorbed photon density (see SI for details). We note that the determination of the photoexcited carrier density is subject to an experimental uncertainty lying in the photon-to-free-charge conversion ratio $\varphi$. The primary origin of this uncertainty is that we cannot entirely neglect the possibility of small fractions of excitons being created rapidly upon photoexcitation, which would lead to the ratio $\varphi$ of the induced charge-carrier density $n$ to the absorbed photon density $\tilde{n}_{0}$ being somewhat lower than unity. On the other hand we use non-resonant excitation conditions with the difference between photon and bandgap energy $(\sim 600 \mathrm{meV})$ being far in excess of plausible binding energies. Therefore photoexcitation is a priori expected to predominantly generate free carriers, since enhancements to the absorption arising from excitons with binding energies of only a few ten meV predominantly occur just below the band gap energy of the semiconductor. ${ }^{33}$

We derive an effective charge-carrier mobility value of $33 \mathrm{~cm}^{2} \mathrm{~V}^{-1} \mathrm{~s}^{-1}$ which represents a lower limit to the chargecarrier mobility $\mu$ (since $0 \leq \varphi \leq 1$ ) and is remarkably high, in agreement with the efficient charge extraction from photovoltaic devices made from such films. ${ }^{4}$ The effective mobility reported here for films of evaporated $\mathrm{CH}_{3} \mathrm{NH}_{3} \mathrm{PbI}_{3-x} \mathrm{Cl}_{x}$ is higher than the previously determined value for the solution-processed material within a mesoporous scaffold $\left(11.6 \mathrm{~cm}^{2} \mathrm{~V}^{-1} \mathrm{~s}^{-1}-\right.$ see ref. $\left.{ }^{7}\right)$, which is most likely the result of the different film morphologies and crystalline domain sizes. ${ }^{45} \mathrm{We}$ note that the value of $\varphi \mu$ determined here for evaporated $\mathrm{CH}_{3} \mathrm{NH}_{3} \mathrm{PbI}_{3-x} \mathrm{Cl}_{x}$ is only a factor of two lower than the value of $\mu$ obtained by means of Hall-effect measurements for annealed polycrystalline pellets of the related $\mathrm{CH}_{3} \mathrm{NH}_{3} \mathrm{PbI}_{3}$. 34 This observation again suggests that the photon-to-freecharge conversion efficiency $\varphi$ is sizeable in these materials, i.e. for the non-resonant excitation used in our experiments, the majority of species generated appear to be free charges, rather than excitons. Therefore free charges seem readily available in these materials following non-resonant photoexcitation, which is highly advantageous for their use in photovoltaic energy conversion.

We continue by performing an analysis of the charge-carrier recombination dynamics that govern the decay of the photoconductivity transients shown in Figure 2. Charge carrier recombination is expected to depend on excitation fluence for simple electron-hole (bimolecular) recombination as well as for higher order decay processes such as Auger recombination in which energy and momentum is transferred to a third charge carrier. ${ }^{35}$ The recombination dynamics of the free charge carrier density $n$ may therefore be described by the following rate equation:

$$
\frac{\mathrm{d} n(t)}{\mathrm{d} t}=-k_{3} n^{3}-k_{2} n^{2}-k_{1} n
$$

where $k_{3}$ is the decay constant describing Auger recombination, $k_{2}$ the bimolecular recombination constant, and $k_{1}$ the rate for monomolecular processes such as trap-assisted charge recombination. Because the measured $\mathrm{THz}$ signal is proportional to the photoinduced conductivity, fitting the above rate equation to the transients actually results in the extraction of the decay constants $k_{3} \varphi^{2}, \mathrm{k}_{2} \varphi$ and $k_{1}$, with the former two representing lower limits to the actual values $k_{2}$ and $k_{3}$ (see ESI). Decay constants were only allowed to vary globally (i.e. had constant values across curves taken at all fluences). The resulting fits are plotted as solid lines in Figure 2 with the extracted values for the effective second- and third-order decay constants given in Table 1. We find a generally low value for the effective bimolecular recombination rate, which is similar to (i.e. $\sim 20 \%$ lower than) the value determined previously for solution-processed $\mathrm{CH}_{3} \mathrm{NH}_{3} \mathrm{PbI}_{3-x} \mathrm{Cl}_{x}$ infiltrated into an inert mesoporous alumina matrix. ${ }^{7}$

\begin{tabular}{lr} 
Material & $\mathrm{CH}_{3} \mathrm{NH}_{3} \mathrm{PbI}_{3-\mathrm{x}} \mathrm{Cl}_{\mathrm{x}}$ \\
\hline Decay constants & \\
$3^{\text {rd }}$ order $\left(\varphi^{2} k_{3}\right)$ & $2.3 \times 10^{-29} \mathrm{~cm}^{6} \mathrm{~s}^{-1}$ \\
$2^{\text {nd }}$ order $\left(\varphi k_{2}\right)$ & $1.1 \times 10^{-10} \mathrm{~cm}^{3} \mathrm{~s}^{-1}$ \\
$1^{\text {st }}$ order $\left(k_{1}\right.$, from ref. $\left.{ }^{30}\right)$ & $1.2 \times 10^{7} \mathrm{~s}^{-1}$ \\
\hline Eff. mobility $(\varphi \mu)$ & $33 \mathrm{~cm}^{2} /(\mathrm{Vs})$ \\
\hline Langevin Ratio & $2 \times 10^{-6} \times \varepsilon$
\end{tabular}

Table 1: Charge-carrier decay rate constants and mobility for vapour-deposited $\mathrm{CH}_{3} \mathrm{NH}_{3} \mathrm{Pbl}_{3-x} \mathrm{Cl}_{x}$. Strictly, the given values for second and third order decay constants, and the effective charge-carrier mobility are lower limits to the actual values of $k_{2}, k_{3}$ and $\mu$ because the photon-to-free-charge conversion efficiency $\phi$ may be lower than unity $(0 \leq \phi \leq 1)$. The 'Langevin Ratio' denotes the ratio between the observed value of $k_{2} / \mu$ to that theoretically predicted by the Langevin model $\left(e /\left(\varepsilon \varepsilon_{0}\right)\right)$. The value stated is exclusive of the factor accounting for the relative permittivity. The static dielectric constant of $\mathrm{CH}_{3} \mathrm{NH}_{3} \mathrm{Pbl}_{3} \quad \varepsilon_{s}=60$ (ref. ${ }^{37}$ ) may serve as an upper limit, since the value of the dielectric function generally decreases with increasing frequency. The mono-molecular decay rate $k_{1}$ was determined from time-resolved photoluminescence measurements as reported in ref. ${ }^{30}$. 
We assess the mono-molecular rate constant $k_{1}$ from the fits to the $\mathrm{THz}$ photoconductivity transients discussed above and find that $k_{1}$ is too low to be accurately determined solely from these data, which is restricted to an experimentally accessible range of pump-probe-delays up to $2.5 \mathrm{~ns}$. From the uncertainty of our fits we can however establish an upper limit for $k_{1}$ of approximately $2 \times 10^{7} \mathrm{~s}^{-1}$. We may further obtain an estimate of the monomolecular charge-carrier decay rate through analysis of the time-resolved photoluminescence (PL) decay, which can straightforwardly be acquired over timescales of hundreds of nanoseconds by means of time-correlated single photon counting. We have recently shown that for low-fluence excitation the PL transients exhibit mono-exponential decay tails ${ }^{30}$ from which we were able to extract a monomolecular rate constant $k_{l}=1.2 \times 10^{7} \mathrm{~s}^{-1}=(83 \mathrm{~ns})^{-1}$. We note that, in general, the dynamics observed with this technique reflect a combination of both excitonic and free charge carrier populations. However, recent observations have suggested that the predominant species present in this material for the low excitation fluences employed in PL measurements are in fact free charges. ${ }^{23}$ This assumption is supported by recent observations of similar decay dynamics observed for both photoinduced absorption and $\mathrm{PL}$ emission in $\mathrm{CH}_{3} \mathrm{NH}_{3} \mathrm{PbI}_{3-x} \mathrm{Cl}_{x}$ films, ${ }^{25}$ and a study of transient microwave conductivity of $\mathrm{CH}_{3} \mathrm{NH}_{3} \mathrm{PbI}_{3}$ on nanosecond-to-microsecond timescales showing coinciding decay lifetimes for photoluminescence and photoconductivity. ${ }^{24}$ The mono-molecular free-charge decay rate for vapour-deposited $\mathrm{CH}_{3} \mathrm{NH}_{3} \mathrm{PbI}_{3-x} \mathrm{Cl}_{x}$ determined through $\mathrm{PL}$ spectroscopy is very low for a polycrystalline material, and only slightly (by about a factor of two) higher than that for solution processed $\mathrm{CH}_{3} \mathrm{NH}_{3} \mathrm{PbI}_{3-x} \mathrm{Cl}_{x}$ infused into a mesoporous alumina matrix. ${ }^{7}$ This result suggests that $\mathrm{CH}_{3} \mathrm{NH}_{3} \mathrm{PbI}_{3-x} \mathrm{Cl}_{x}$ has a generally low propensity to trap free charge carriers, regardless of the general processing route taken.

For a quantitative discussion of the obtained electron-hole recombination rate we compare our results to the prediction of Langevin theory. The Langevin model is purely kinetic approach towards calculating the bimolecular decay constant and assumes that once an electron and a hole move within their joint Coulomb capture radius, recombination will occur. ${ }^{38} 39$ The predicted bimolecular decay constant, $\mathrm{k}_{2, \mathrm{~L}}=\mu e\left(\varepsilon_{0} \varepsilon_{\mathrm{r}}\right)^{-1}$ is found to be proportional to the charge carrier mobility. Therefore, recombination governed by the Langevin model imposes a fundamental limit to the operation of photovoltaic devices, which require long carrier diffusion lengths and hence both low charge recombination rates and high mobilities. However, we have recently shown that solution-processed organolead halide perovskites defy this limit, with the actual ratio of bimolecular charge recombination rate to charge mobility lying about five orders of magnitude below that predicted by the Langevin model. ${ }^{7}$ For the vapour-deposited films of $\mathrm{CH}_{3} \mathrm{NH}_{3} \mathrm{PbI}_{3-x} \mathrm{Cl}_{x}$ investigated here, we find that the ratio $\varphi k_{2} /(\varphi \mu)$ again lies below the value of $e\left(\varepsilon_{0} \varepsilon_{\mathrm{r}}\right)^{-1}$ expected from Langevin theory by about five orders of magnitude (see Table 1). These materials therefore fundamentally appear to exhibit particular mechanisms inhibiting electron-hole recombination, independent of the deposition route. Several other active materials for solar cells, such as de-mixed blends of conjugated polymer with fullerene derivatives 4041 or amorphous silicon, ${ }^{42}$ have in the past been found to exhibit non-Langevin recombination, which was in both cases attributed to factors causing spatial separation of the electron- hole pair. As we suggested recently, polarization of free-charge pairs across the metal-halide bond may be a cause of stabilization for a spatially charge-separated state. ${ }^{7}$ Such effects have been intensively examined for a range of ionic solids such as alkali halides, ${ }^{31}$ for which the valence band has strong halide character (i.e. localizing the hole in the halide vicinity), and they are also apparent in recent density-functional calculations for $\mathrm{CsPbI}_{3}$ perovskites. ${ }^{43}$ Alternatively, more extended electron-hole spatial separations may be induced through builtin electric fields. Many materials in perovskite structures with lack of inversion symmetry display ferroelectric effects, ${ }^{44} 45$ allowing such fields to be present even in the bulk material. Direct experimental assessments of the extent to which $\mathrm{CH}_{3} \mathrm{NH}_{3} \mathrm{PbI}_{3-x} \mathrm{Cl}_{x}$ and related compounds exhibit ferroelectricity is still scarce. However, recent density functional theory studies have indicated that such hybrid perovskites are expected to exhibit spontaneous electric polarization. ${ }^{46} 47$ Such effects could aid photovoltaics performance by facilitating charge separation and by inhibiting subsequent recombination. ${ }^{45}$ They may however also lead to a dependence of photovoltaic performance on ferroelectric domain size, and hysteresis effects in the cells' current-voltage characteristics. ${ }^{48}$ An understanding of the mechanisms for the observed strongly non-Langevin electron-hole recombination dynamics would allow design rules to be established for a wider class of materials for photovoltaics.

With knowledge of charge carrier mobility and lifetimes in $\mathrm{CH}_{3} \mathrm{NH}_{3} \mathrm{PbI}_{3-x} \mathrm{Cl}_{x}$ we may calculate the charge diffusion length, which is central to the performance of absorber layers in solar cells. Especially in planar heterojunction device architectures, charge-carrier diffusion lengths are required that sufficiently exceed the thickness of the active layer (currently $\sim 330 \mathrm{~nm}$ in planar heterojunction $\mathrm{CH}_{3} \mathrm{NH}_{3} \mathrm{PbI}_{3-x} \mathrm{Cl}_{x}$ based cells ${ }^{4}$ ) to achieve satisfactory current densities under working conditions where no electric field is present. In the presence of bimolecular and Auger recombination processes, the diffusion length $L_{D}$ depends on carrier density $n$ through

$$
L_{D}(n)=\sqrt{\frac{D}{R_{\text {total }}(n)}}
$$

where $D=\mu k_{B} T e^{-1}$ is the diffusion constant at room temperature, and $R_{\text {total }}$ the total recombination rate

$$
R_{\mathrm{total}}=-\frac{1}{n} \frac{\mathrm{d} n}{\mathrm{~d} t}=n^{2} k_{3}+n k_{2}+k_{1}
$$

We evaluate $L_{D}$ as a function of $n$, using the determined recombination rate constants and charge mobility listed in Table 1. The resulting plots are shown in Figure 3 for which we assumed a photon-to-free-charge conversion ratio $\varphi=1$ for simplicity. We find that for charge-carrier concentrations covering realistic solar cell operating conditions, the diffusion length in $\mathrm{CH}_{3} \mathrm{NH}_{3} \mathrm{PbI}_{3-x} \mathrm{Cl}_{x}$ is on the order of microns $(2.7 \mu \mathrm{m}$ at $n=10^{15} \mathrm{~cm}^{-3}$ ) and is only limited by mono-molecular decay processes. At higher concentrations $\left(n \geq 10^{18} \mathrm{~cm}^{-3}\right)$ bimolecular and finally Auger recombination reduce the diffusion length to the sub-micron range. We note that these diffusion lengths are probed at $\mathrm{THz}$ frequencies causing only short-range carrier displacement and thus the long-range DC values would be somewhat shorter, if the charge motion was strongly inhibited at grain boundaries. However, recent electron-beam induced current (EBIC) measurements have suggested that while for 
solution-processed $\mathrm{CH}_{3} \mathrm{NH}_{3} \mathrm{PbI}_{3}$ such effects may occur (in particular for electrons), solution-processed $\mathrm{CH}_{3} \mathrm{NH}_{3} \mathrm{PbI}_{3-x} \mathrm{Cl}_{x}$ layers allow for largely uninhibited electron and hole extraction with diffusion lengths approaching 2 microns. ${ }^{49} 50$ Our observation of $\mathrm{THz}$ spectra exhibiting Drude-like free carrier response demonstrates that scattering from grain barriers appears to be largely absent also in vapour-deposited $\mathrm{CH}_{3} \mathrm{NH}_{3} \mathrm{PbI}_{3-x} \mathrm{Cl}_{x}$. The extracted diffusion lengths hence represent a realistic value of what out to be achievable in thin films with typically large crystallite size.

In addition, Figure 3 also shows the diffusion lengths expected for the two hypothetical cases of (i) solely kinetically limited bi-molecular recombination rates (Langevin recombination, $\left.k_{2}=\mu e\left(\varepsilon_{0} \varepsilon_{\mathrm{r}}\right)^{-1}\right)$, and for (ii) complete absence of mono-molecular decay channels $\left(k_{1}=0\right)$. The plot shows that the situation would change dramatically if the material were to exhibit Langevin-recombination (case (i)). Here, bimolecular recombination would dominate through the entire density range shown in the plot limiting the diffusion length to a few tens of nanometers at a carrier concentration of $10^{15} \mathrm{~cm}^{-3}$. Without the observed non-Langevin decay mechanisms, these materials would therefore only find application as thin sensitizer layers on nano- or meso-structured electron- and hole-extracting layers, such as those employed in dye-sensitized solar cells. For case (ii) on the other hand, the elimination of trap-mediated decay channels would enhance the diffusion length significantly $\left(29 \mu \mathrm{m}\right.$ at $\left.n=10^{15} \mathrm{~cm}^{-3}\right)$ and make it dependent on absorbed photon flux over the typical solar cell operating range. We note that previously reported values of $k_{1}$ for solution processed films ${ }^{257}$ are lower (e.g. $k_{1}=5 \times 10^{6} \mathrm{~s}^{-1}-$ see ref. ${ }^{7}$ ) than the mono-molecular rate we measured for vapourdeposited films. This comparison suggests that the monomolecular charge recombination rate is governed at least to some extent by defects or impurities in the bulk and at grain boundaries, which can be targeted by careful engineering of the processing route. In this respect, improvements of the vapour deposition protocol could very well hold the potential for solar cells showing even higher photocurrent and power conversion efficiencies.

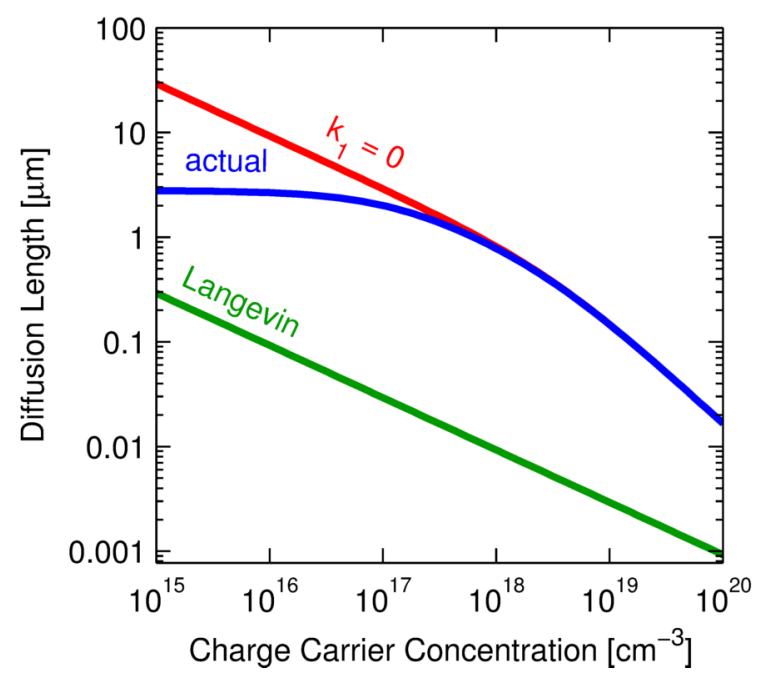

Figure 3: Charge-carrier diffusion lengths as a function of charge-carrier density, calculated from the decay rate constants in Table 1, assuming a photon-to-free-charge conversion efficiency $\phi=1$. The blue line shows the actual diffusion length to be expected from the measured first-, second- and third-order rates. The red and green lines show hypothetical values for the two cases of (i) green: bi-molecular recombination rates instead being governed by Langevin recombination, i.e. $k_{2}=\mu e\left(\varepsilon_{0} \varepsilon_{\mathrm{r}}\right)^{-1}$, taking the low-frequency limit of $\varepsilon_{\mathrm{r}}=60$ (ref. ${ }^{37}$ ), and for (ii) red: complete absence of mono-molecular decay channels $\left(k_{1}=0\right)$.

\section{Conclusions}

We have investigated two key properties for photovoltaic operation, charge carrier mobility and lifetime, for vapour-deposited films of the organolead halide perovskite $\mathrm{CH}_{3} \mathrm{NH}_{3} \mathrm{PbI}_{3-x} \mathrm{Cl}_{x}$. The high-quality films obtained through dual-source evaporation show excellent charge carrier mobility of at least $33 \mathrm{~cm}^{2} \mathrm{~V}^{-1} \mathrm{~s}^{-1}$, and low bi-molecular recombination rates that defy the prediction of the Langevin model by about five orders of magnitude. As a result, the diffusion lengths are only limited by mono-molecular decay processes under realistic solar cell operating conditions. We find that the extracted long diffusion lengths approaching 3 microns result from the combination of both non-Langevin bimolecular electronhole recombination, and exceptionally low trap-induced monomolecular charge recombination pathways. In comparison to our recent study of solution-processed films of $\mathrm{CH}_{3} \mathrm{NH}_{3} \mathrm{PbI}_{3-x} \mathrm{Cl}_{x}$ infused into an alumina matrix, ${ }^{7}$ we find that vapour-deposited films exhibit higher charge-carrier mobilities and monomolecular recombination rates, but similar bimolecular recombination. This change in the former two parameters coincidentally cancels when the diffusion lengths are evaluated, leading to similar calculated values of $L_{D}$ for the two processing methods. Comparison of parameters obtained for the two deposition routes suggests that while the non-Langevin bimolecular decay rates are an inherent property of this material, charge carrier mobilities and trap densities may vary with processing conditions. Finding ways to identify and eliminate traps, and to enlarge crystallite size further, would therefore allow further increases in photovoltaic device efficiencies. In addition, there is a clear need for finding an explanation for the underlying mechanism causing the observed non-Langevin charge recombination. This mechanism could also be to some extent responsible for the large fraction of initially generated free-charge carriers that appear to be present following light absorption under a wide range of excitation conditions. Such an understanding would greatly aid the development of new material classes that not only exhibit excellent photovoltaic performance, but also have additional benefits such as higher long-term stability or lead-free composition.

\section{Acknowledgements}

The authors gratefully acknowledge funding from the Engineering and Physical Sciences Research Council.

\section{Notes and references}

University of Oxford, Department of Physics, Clarendon Laboratory, Parks Road, Oxford, OX1 3PU, United Kingdom. E-mail: 1.herz@physics.ox.ac.uk

$\dagger$ Electronic Supplementary Information (ESI) available: Includes experimental methods, SEM images, details of the derivation of the $\mathrm{THz}$ mobility and fits of $\mathrm{THz}$ photoconductivity transients and spectra. See DOI: $10.1039 / \mathrm{c} 000000 \mathrm{x} /$

1. B. W. D'Andrade and S. R. Forrest, Adv. Mater., 2004, 16, 
1585-1595.

2. S. Forrest, Nature, 2004, 428, 911-918.

3. S. Bae, H. Kim, Y. Lee, X. Xu, J.-S. Park, Y. Zheng, J. Balakrishnan, T. Lei, H. Ri Kim, Y. I. Song, Y.-J. Kim, K. S. Kim, B. Ozyilmaz, J.-H. Ahn, B. H. Hong and S. Iijima, Nat Nano, 2010, 5, 574-578.

4. M. Liu, M. B. Johnston and H. J. Snaith, Nature, 2013, 501, 395-398.

5. J. M. Ball, M. M. Lee, A. Hey and H. J. Snaith, Energy Environ. Sci., 2013, 6, 1739-1743.

6. M. Riede, C. Uhrich, J. Widmer, R. Timmreck, D Wynands, G. Schwartz, W.-M. Gnehr, D. Hildebrandt, A. Weiss, J. Hwang, S. Sundarraj, P. Erk, M. Pfeiffer and K. Leo, Adv. Funct. Mater., 2011, 21, 3019-3028.

7. C. Wehrenfennig, G. E. Eperon, M. B. Johnston, H. J. Snaith and L. M. Herz, Adv. Mater., 2014, 26, 1584-1589.

8. M. M. Lee, J. Teuscher, T. Miyasaka, T. N. Murakami and H. J. Snaith, Science, 2012, 338, 643-647.

9. H.-S. Kim, C.-R. Lee, J.-H. Im, K.-B. Lee, T. Moehl, A. Marchioro, S.-J. Moon, R. Humphry-Baker, J.-H. Yum, J. E. Moser, M. Gratzel and N.-G. Park, Sci. Rep., 2012, 2, 591.

10. H. J. Snaith, J. Phys. Chem. Lett., 2013, 4, 3623-3630.

11. H. S. Kim, S. H. Im and N.-G. Park, J. Phys. Chem. C, 2014, 118, 5615-5625.

12. G. E. Eperon, V. M. Burlakov, P. Docampo, A. Goriely and H. J. Snaith, Adv. Funct. Mater., 2014, 24, 151-157.

13. A. Dualeh, N. Tétreault, T. Moehl, P. Gao, M. K Nazeeruddin and M. Grätzel, Adv. Funct. Mater., 2014, in press, DOI: $10.1002 /$ adfm.201304022.

14. E. Mosconi, A. Amat, M. K. Nazeeruddin, M. Grätzel and F. De Angelis, J. Phys. Chem. C, 2013, 117, 13902-13913.

15. B. Conings, L. Baeten, C. De Dobbelaere, J. D'Haen, J. Manca and H.-G. Boyen, Adv. Mater., 2013, 26, 20412046.

16. C. A. Schmuttenmaer, Chem. Rev., 2004, 104, 1759-1779.

17. J. Lloyd-Hughes and T.-I. Jeon, J. Infrared. Millim. Te., 2012, 33, 871-925.

18. H. Němec, P. Kužel and V. Sundström, J. Photochem. Photobiol. A-Chem., 2010, 215, 123-139.

19. R. Ulbricht, E. Hendry, J. Shan, T. F. Heinz and M. Bonn, Rev. Mod. Phys., 2011, 83, 543-586.

20. M. Hirasawa, T. Ishihara, T. Goto, K. Uchida and N. Miura, Physica B, 1994, 201, 427-430.

21. K. Tanaka, T. Takahashi, T. Ban, T. Kondo, K. Uchida and N. Miura, Solid State Commun., 2003, 127, 619-623.

22. S. Sun, T. Salim, N. Mathews, M. Duchamp, C. Boothroyd, G. Xing, T. C. Sum and Y. M. Lam, Energy Environ. Sci., 2014, 7, 339-407.

23. V. D'Innocenzo, G. Grancini, M. J. P. Alcocer, A. R. S. Kandada, S. D. Stranks, M. M. Lee, G. Lanzani, H. J. Snaith and A. Petrozza, Nat. Commun., 2014, 5, 3586.

24. A. Marchioro, J. Teuscher, D. Friedrich, M. Kunst, R. van de Krol, T. Moehl, M. Gratzel and J.-E. Moser, Nat. Photon., 2014, 8, 250-255.

25. S. D. Stranks, G. E. Eperon, G. Grancini, C. Menelaou, M. J. P. Alcocer, T. Leijtens, L. M. Herz, A. Petrozza and H. J.
Snaith, Science, 2013, 342, 341-344.

26. N. V. Smith, Phys. Rev. B, 2001, 64, 155106.

27. H. Němec, P. Kužel and V. Sundström, Phys. Rev. B, 2009, 79, 115309.

28. A. Maalej, Y. Abid, A. Kallel, A. Daoud, A. Lautié and F. Romain, Solid State Communications, 1997, 103, 279-284.

29. C. Quarti, G. Grancini, E. Mosconi, P. Bruno, J. M. Ball, M. M. Lee, H. J. Snaith, A. Petrozza and F. D. Angelis, J. Phys. Chem. Lett., 2014, 5, 279-284.

30. C. Wehrenfennig, M. Liu, H. J. Snaith, M. B. Johnston and L. M. Herz, J. Phys. Chem. Lett., 2014, 5, 1300-1306.

31. R. T. Williams and K. S. Song, J. Phys. Chem. Solids, 1990, 51, 679-716.

32. H.-K. Nienhuys and V. Sundström, Phys. Rev. B, 2005, 71, 235110 .

33. M. Fox, Optical Properties of Solids, 2nd ed., Oxford University Press, New York, 2010.

34. C. C. Stoumpos, C. D. Malliakas and M. G. Kanatzidis, Inorg. Chem., 2013, 52, 9019-9038.

35. A. R. Beattie and P. T. Landsberg, P. Roy. Soc. Lond. A Mat., 1959, 249, 16-29.

36. A. Haug, J. Phys. Chem. Solids, 1988, 49, 599-605.

37. N. Onoda-Yamamuro, T. Matsuo and H. Suga, J. Phys. Chem. Solids, 1992, 53, 935-939.

38. P. Langevin, Ann. Chim. Phys., 1903, 28, 433-530.

39. M. Pope and C. E. Swenberg, Electronic Processes in Organic Crystals and Polymers; Oxford Science, New York, 1999.

40. P. Parkinson, J. Lloyd-Hughes, M. B. Johnston and L. M. Herz, Phys. Rev. B, 2008, 78, 115321.

41. A. Pivrikas, G. Juška, A. J. Mozer, M. Scharber, K. Arlauskas, N. S. Sariciftci, H. Stubb and R. Österbacka, Phys. Rev. Lett., 2005, 94, 176806.

42. G. J. Adriaenssens and V. I. Arkhipov, Solid State Com., 1997, 103, 541-543.

43. J. Even, L. Pedesseau, J.-M. Jancu and C. Katan, J. Phys. Chem. Lett., 2013, 4, 2999-3005.

44. T. Choi, S. Lee, Y. J. Choi, V. Kiryukhin and S.-W. Cheong, Science, 2009, 324, 63-66.

45. I. Grinberg, D. V. West, M. Torres, G. Gou, D. M. Stein, L. Wu, G. Chen, E. M. Gallo, A. R. Akbashev, P. K. Davies, J. E. Spanier and A. M. Rappe, Nature, 2013, 503, 509-512.

46. F. Brivio, A. B. Walker and A. Walsh, APL Mater., 2013, 1, 042111.

47. J. M. Frost, K. T. Butler, F. Brivio, C. H. Hendon, M. van Schilfgaarde and A. Walsh, Nano Lett., 2014, in press, DOI: $10.1021 / \mathrm{n} 1500390 f$.

48. H. J. Snaith, A. Abate, J. M. Ball, G. E. Eperon, T. Leijtens, N. K. Noel, S. D. Stranks, J. T.-W. Wang, K. Wojciechowski and W. Zhang, J. Phys. Chem. Lett., 2014, 5, 1511-1515.

49. E. Edri, S. Kirmayer, A. Henning, S. Mukhopadhyay, K. Gartsman, Y. Rosenwaks, G. Hodes and D. Cahen, Nano Lett., 2014, 14, 1000-1004.

50. E. Edri, S. Kirmayer, S. Mukhopadhyay, K. Gartsman, G. Hodes and D. Cahen, Nature Comm., 2014, 5, 3461. 